\title{
Orientational anisotropy in the human visual system
}

\author{
BILL JENKINS \\ University of Western Australia, Nedlands, Australia
}

\begin{abstract}
A global pattern/randomness threshold was used to investigate orientational anisotropy in the human visual system. Dynamic transpositionally symmetric textures were used, since they allowed the investigation of three theoretically derived variables, size, orientational uniformity, and numerosity, independently and in interaction. The detectability of correlation, as indexed by these thresholds, was found to be dependent on the size of the uniform point-pair elements, $\Delta d$, and their orientation; larger tolerable $\Delta d$ values were found in the vertical meridian. Increasing the stimulus field size increased the absolute value of this threshold, but not the relative value as a function of orientation. The thresholds were also found to be dependent on the orientational uniformity of the point-pairs. Tolerable perturbation did not vary as a function of the uniform orientation until a critical point-pair size was exceeded. This critical point-pair size did vary as a function of orientation, falling quite distinctly from vertical, to horizontal, to oblique. The number of point-pair elements comprising the textures did not affect the global pattern/randomness threshold. The implications of these data for the neural mechanisms underlying the "oblique effect" were examined, and it was concluded that an orientational anisotropy might exist in the length of receptive fields serving functional units selectively sensitive to orientation. It was also concluded, however, that this factor alone could not account for the various examples of the orientational anisotropy in vision, and that any anisotropy measured in human vision might be the net product of more than one anisotropy existing within and between the various levels of processing in the human visual system.
\end{abstract}

Orientation selectivity is a major feature of the functional architecture of the macaque monkey visual cortex (Hubel \& Wiesel, 1977). Evidence for a similar specificity in the human visual system has emerged from several different sources: from visual masking (e.g., Blakemore, Nachmias, \& Sutton, 1970; Campbell \& Kulikowski, 1966; Houlihan \& Sekuler, 1968; Parlee, 1969; Sekuler, 1965), adaptation studies (e.g., Blakemore \& Nachmias, 1971; Gibson, 1933; Gilinsky \& Mayo, 1971; Movshon \& Blakemore, 1973), and induction and aftereffects (e.g., Blakemore, Carpenter, \& Georgeson, 1970; Campbell \& Maffei, 1970).

The reductive logic underlying these investigations is the indirect argument that if stimulus A and stimulus B interact psychophysically, but stimulus $\mathrm{A}$ and stimulus $\mathrm{C}$ do not, then a structure or process exists that is common to $\mathrm{A}$ and $\mathrm{B}$ but not to $\mathrm{C}$ (see Braddick, Campbell, \& Atkinson, 1978). A different behavioral argument has been used by Sutherland (1957) to separate structures on the basis of the capacity to discriminate: if an organism is capable of discriminating between two stimuli on a single stimulus dimension, then, given no artifactual con-

This research was supported by a grant from the Australian Research Grants Scheme to the author (ARGS Grant A28315129). H. Jenkins is thanked for her research assistance. The referees' comments were also of great value.

The author's mailing address is: Department of Psychology, University of Western Australia, Nedlands, W.A. 6009, Australia. founding, it must possess mechanisms that are selectively sensitive along that dimension. One further argument can be made to support the existence of independent neural structures in the human visual system: if we are differentially sensitive to particular values on any one stimulus dimension, then it is probable that separate processes or structures exist that reflect this differential sensitivity.

In the case of orientational selectivity, the anisotropy is well documented and ubiquitous (Appelle, 1972). In humans, there appears to be a difference in sensitivity between the horizontal, vertical, and oblique orientations at the retina, with the visual system being less sensitive to the latter. It is found in diverse areas: in symmetry detection (e.g., Jenkins, 1983a; Julesz, 1971), in pattern reversal (e.g., Kitterle, Russell, \& Nixon, 1974), in the extent of illusions (e.g., Anton, 1976), in acuity (e.g., Emsley, 1925; Leibowitz, 1955), in adjusting lines to specific angles (e.g., Jastrow, 1892), in detecting embedded figures (e.g., Appelle, 1976), in afterimage visibility (e.g., Atkinson, 1972), and in judgments of parallelness (e.g., Rochlin, 1955). Orientational anistropy has also been reported in studies of visual masking, adaptation, and aftereffects (e.g., Campbell \& Kulikowski, 1966; Campbell, Kulikowski, \& Levinson, 1966; Furchner \& Young, 1975; Green \& Hoyle, 1964; Jastrow, 1892; Kohler \& Wallach, 1944).

Explanations to account for this orientational anisotropy are many and varied. Emsley (1925) proposed that the differential sensitivity was due to astigmatism, but revised 
his opinion when he corrected for this factor and found that the anisotropy remained. His ambivalence towards this finding is mirrored in his term "residual astigmatism." Any attempt to account for the anisotropy by referring to the optics of the eye has to be discarded on the basis of investigations that bypass the dioptrics (e.g., Freeman \& Thibos, 1973; Maffei \& Campbell, 1970). In addition, the suggestion that the orientational anisotropy is the result of interaction with the environment (e.g., Annis \& Frost, 1973; Gregory, 1972) is unsatisfactory, since it does nothing to spell out the nature of the interaction or its locus.

More productively, Atkinson (1972) has put forward three possible explanations for the anisotropy in terms of attributes of functional units at the cortical level. Her suggestions are: (1) that vertical and horizontal units have longer receptive fields than oblique units (a "size" hypothesis); (2) that vertical and horizontal units are more finely tuned, with a higher selectivity for orientation than oblique units (a "selectivity"' hypothesis); (3) that units serving the oblique orientation are less numerous than those serving other more perceptible orientations (a "numerosity" hypothesis).

It should be noted that these hypotheses are not mutually exclusive, neither are they directly testable using psychophysical techniques. Yet each identifies a single stimulus dimension that can be examined psychophysically.

The aim of this investigation was to determine whether the orientational anisotropy existed with respect to any of the three derivative dimensions of size, orientational uniformity, and numerosity, using a stimulus that could be manipulated along these dimensions.

Dynamic transpositionally symmetric textures allow the necessary manipulations, since they comprise the sequential plotting of identical point-pairs randomly in space and uniformly over time. They have been used in other investigations to map spatial and temporal limits to the detection of correlation (Jenkins, 1983b), the detection of bilateral symmetry (Jenkins, 1982, 1983a), and the spatial and temporal tolerance for the detection of pattern and motion (Jenkins, 1983c; Morgan \& Ward, 1980). The use of dynamic textures allows the investigation of both spatial and temporal attributes of the visual system; but more importantly to the specific aim of this investigation, these textures allow the simultaneous manipulation of the number of stimulus elements presented within a given area at the retina, their orientational uniformity, and their size.

The value of using dynamic point-pair textures to investigate these variables is also enhanced by theoretical speculation (Glass, 1969; Glass \& Perez, 1973; Glass \& Switkes, 1976; Jenkins, 1983b) that the neural structures involved in the detection of structure in transpositionally symmetric textures comprise functional units at the cortical level that can be activated by point-pair elements. So, although direct evaluation of Atkinson's hypotheses is not possible psychophysically, the experimental results may provide the basis for further speculation.

\section{GENERAL METHOD}

The following description is an account of the methods that were common to all the experiments reported.

\section{Experimental Situation}

All experiments utilized dynamic dot textures plotted on a display oscilloscope under computer control. Subjects sat viewing the oscilloscope binocularly, at a distance of $57.3 \mathrm{~cm}$. The diameter of the circular dot field subtended $5^{\circ}$ of visual angle (5-cm diameter), and the viewing distance was maintained by the use of a chin-andforehead restraint for the subjects.

There was sufficient illumination for the subject to see the face of the oscilloscope, so that convergence and accommodation could be maintained at the appropriate distance. The luminance of the oscilloscope face, without stimulus, was $1.0 \mathrm{~cd} / \mathrm{m}^{2}$.

Illumination of the oscilloscope face was provided from the front by a Carousel projector with a filtered lens that approximated the color of the dots comprising the dot fields. The dynamic stimulus field luminance was maintained at $1.2 \mathrm{log}$ units above threshold throughout all experiments. For all experiments, the laboratory was dimly illuminated, so that the subjects did not undergo any radical change in visual sensitivity while waiting to participate in the experiments.

\section{Apparatus}

The experiments were run under the control of a PDP-8/E computer, manufactured by the Digital Equipment Corporation. Interfaced to this computer was a fast display oscilloscope for presentation of the stimuli, a Hewlett-Packard 1332A oscilloscope equipped with an ultrashort P24 phosphor. The intensity of a point plotted on this type of phosphor has a single peak with a maximum spectral energy emission at $510 \mathrm{~nm}$, and fades to $10 \%$ of its original brightness in $1.5 \mu \mathrm{sec}$. The effective physical duration of each point is therefore taken to be approximately $1.5 \mu \mathrm{sec}$.

\section{Generation of Stimuli}

The dynamic dot fields were plotted on an oscilloscope face within a square $9 \times 9 \mathrm{~cm}$ region. This region was a $256 \times 256$ matrix, so that the location of any point was given by one of 256 possible $x$ values and one of 256 possible $y$ values.

A single random point could be plotted by obtaining a random number between 0 and 255 for $x$ and, similarly, a random number for $y$. The numbers were obtained using the computer's randomnumber generator. These two coordinates, together with an intensity value, were then inserted into the appropriate locations in a computer plotting subroutine. The intensity value was held constant during all experiments. Once these values had been inserted, the next section in the program plotted the point at the specified coordinates on the oscilloscope screen.

The psychophysical method used was a two-alternative forced choice; the alternatives were a random-dot texture and a point-pair texture constructed as described below:

Dynamic random dot textures. These were constructed by the sequential plotting of random points, where each point was independent of any other point. The points were plotted at the rate of one every $60.8 \mu \mathrm{sec}$. Since the total duration of the stimulus field was $1 \mathrm{sec}$, the computer plotted 16,446 points. However, the number of points actually seen by the observer was subject to temporal and spatial constraints.

The temporal constraint was provided by the observer himself, with his limited integration period, which is approximately $130 \mathrm{msec}$ for dynamic visual noise of the intensity used. At any one instant, he could see only the number of points that were plotted within this period (approximately 2,138 points). He could, of course, see this many points for the full stimulus duration of $1 \mathrm{sec}$.

The spatial constraint was imposed externally, and was given by the fact that the area of $9 \times 9 \mathrm{~cm}$ was masked, with the exception 
of a circular region concentric with the square and with a radius of $2.5 \mathrm{~cm}$. The area of such a circle is $19.64 \mathrm{~cm}^{2}$. So the proportion of dots seen within this circular area was $19.64 / 81=0.242$; and therefore the average number of dots seen was $2,138 \times 0.242$ $=517$ points, which corresponds to a density of $517 / 19.64=26$ points $/ \mathrm{cm}^{2}$ (or 26 points per square degree of visual angle, given the viewing distance of $57.3 \mathrm{~cm}$ ).

Dynamic random point-pair textures. These fields were constructed by the sequential plotting of identical point-pairs. Each point-pair was determined in the same manner: after the $x$ and $y$ values of the origin point were determined randomly, the $y$ value was incremented by a constant for vertical point-pairs. The new $y$ value, together with the original $x$ value, specified the coordinates of the partner point. Both points were then plotted on the oscilloscope screen (within a period of $5 \mu \mathrm{sec}$ ). The increment in all the experiments is specified as within point-pair separation, $\Delta d$, and is measured in degrees of visual angle.

The point-pairs were plotted at a rate of one pair every $121.6 \mu \mathrm{sec}$. To obtain a stimulus duration of $1 \mathrm{sec}$, this meant that 8,223 pointpairs were plotted by the computer. As in the case of the randomdot fields, however, the temporal integration period of approximately $130 \mathrm{msec}$ and the 5 -cm-diam mask resulted in approximately 258 point-pairs' being perceptually available, providing an average density of approximately 26 points per square degree of visual angle, the same as for the random-dot field.

Given the circularity of the stimulus texture, the various pointpair orientations, vertical, horizontal, and oblique, were obtained simply by rotating the oscilloscope to the appropriate orientation. Because pilot measurements revealed no difference between obliques of $45^{\circ}$ or $135^{\circ}$ only $45^{\circ}$ data are reported.

\section{Experimental Procedure}

The psychophysical method used was a temporal two-alternative forced-choice technique, where one stimulus comprised point-pairs and the other an equally dense field of random points. On each trial the presentation order of the two fields was random and the subject was required to identify and signal the temporal location of the point-pair stimulus. If the subject was unsure, he was instructed to guess.

Each session comprised 100 trials, 20 trials of each of five values of the independent variable presented randomly. The order of stimulus location within each trial was also random. Five sessions were completed by each subject for each experimental condition.

To initiate a trial, the subject depressed one of the two handheld pushbuttons, and the stimulus sequence commenced immediately. Two dot fields appeared on the screen sequentially, each of $1 \mathrm{sec}$ duration and both separated by a 1-sec interstimulus interval. The subject then identified the temporal location of the point-pair stimulus and pressed the pushbutton corresponding to "first" or "second," as appropriate. This decision was signaled to the computer, and display parameters were then computed for the next trial (computing time, approximately $10 \mathrm{msec}$ ); the subject was then free to initiate the next trial. There was no pressure to complete a session within a given time limit, but in practice it was found that each set of 100 trials was completed in approximately $15 \mathrm{~min}$. At the conclusion of each session, the computer printed out the results on the teletypewriter.

\section{Subjects}

The sequence of events outlined in the preceding section was conducted with three observers, all of whom had normal or correctedto-normal vision.

\section{Correlation Detection Limit}

As the within-point-pair separation $(\Delta \mathrm{d})$ is increased or the orientational uniformity $(\Delta p)$ perturbed, the global pattern of striation apparent in these transpositionally symmetric textures (see Figure 1) disappears, to be replaced by perceived randomness. The threshold between these two perceptual states, global pattern and perceived randomness, is considered to be the limit for correlation detection and is calculated in the classical manner; that is, the threshold is the value of $\Delta \mathrm{d}$ or $\Delta \mathrm{p}$ that falls at the midpoint between perfect discrimination and chance, when a manipulated symmetric texture is compared with an equally dense random-dot texture.

\section{EXPERIMENT 1: ORIENTATIONAL ANISOTROPY AND SIZE}

If a dynamic transpositionally symmetric texture is constructed by the rapid plotting of identical point-pairs, uniform in size $(\Delta d)$ and uniform in orientation, and if $\Delta d$ is within a measured spatial limit (Jenkins, 1983b), the visual system can detect correlation. The perceptual resultant corresponds in this case to coherent global pattern (see Figure 1, upper). If $\Delta d$ is increased beyond this spatial limit, then the detection of correlation breaks down and the perceptual result is perceived randomness (see Figure 1, lower). In other words, the threshold between perceiving global pattern and perceiving randomness
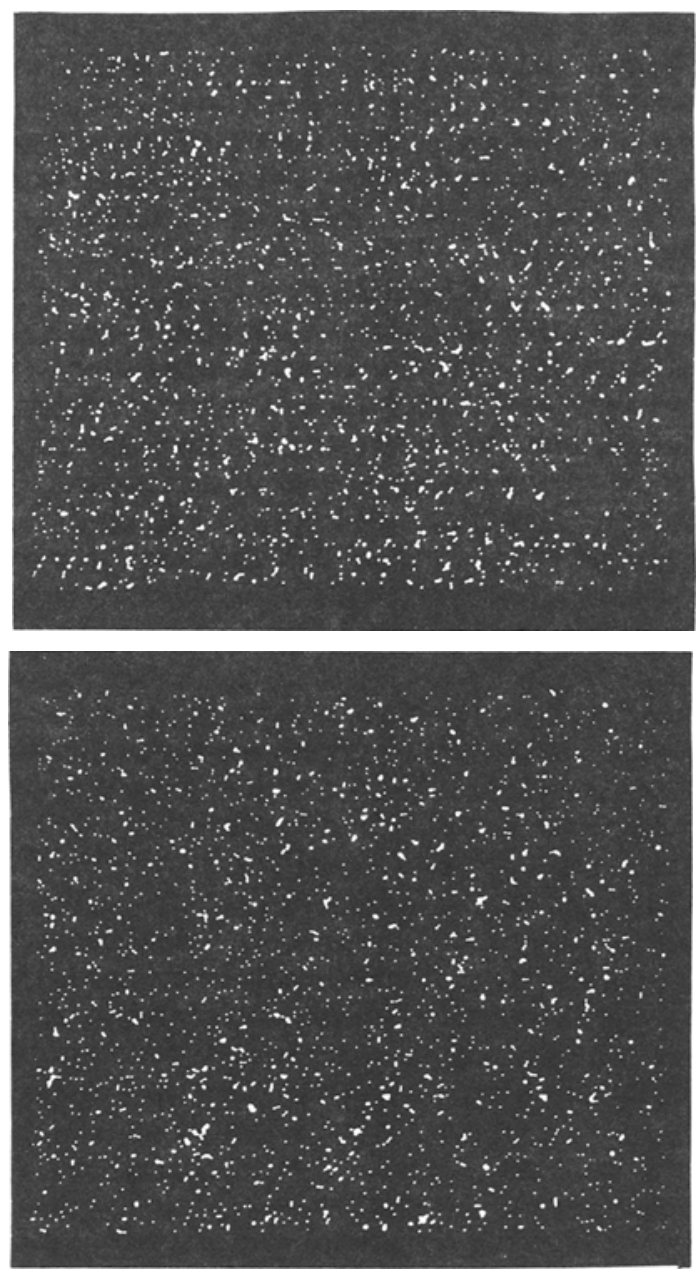

Figure 1. A static form of transpositionally symmetric texture, horizontally oriented, with $\Delta d$ (within-point-pair separation) within the correlation detection limit (upper) and outside this detection limit (lower). 
could be said to represent the spatial limit of the correlational mechanism of the human visual system.

The aim of Experiment 1 was to measure the spatial limit for three orientations. In addition, this limit was measured with three different stimulus field sizes, since this factor is known to correlate with the greatest variation in the spatial limit (Jenkins, 1983b). If variation in the spatial limit is obtained as a function of orientation and this variation is maintained over a range of stimulus sizes, then an orientational anisotropy could be said to exist in the detection of correlation.

\section{Method}

The aim of Experiment 1 was to measure the spatial limit to the detection of correlation, using transpositionally symmetric dynamic textures, for three orientations, vertical, horizontal, and oblique, and for three stimulus field sizes, with diameters of $2.5^{\circ}, 5.0^{\circ}$, and $7.5^{\circ}$ of visual angle.

The variation in orientation was attained by rotating the display oscilloscope; field size variation was achieved by masking the oscilloscope face with the exception of a circular region concentric with the square face, with diameters of $2.5,5.0$ and $7.5 \mathrm{~cm}$., depending on the field size required. Additional detail can be found in the general method section.

The extent of transposition was varied in one stimulus and compared with an equally dense random-dot texture, using a twoalternative, forced-choice technique. The subject's task was to identify the temporal location of the nonrandom stimulus.

\section{Results and Discussion}

Figure 2 illustrates the individual psychometric functions for three subjects for a stimulus field diameter of $5^{\circ}$ of visual angle. They all show that discrimination between the point-pair texture and the equally dense random texture breaks down as $\Delta \mathrm{d}$ is increased. These functions also show that detection of correlation in the symmetric texture is superior when the uniformly oriented point-pairs are vertical. There appears to be no consistent distinction between horizontal and oblique.

The effect of stimulus field size is shown in Figure 3, where it can be seen that as the field diameter increases, so too does the spatial limit. The larger spatial limit for vertical point-pair textures is evident at all field sizes, for all subjects. There again appears to be no systematic difference between horizontal and oblique spatial limits. It is not possible to determine the relationship between the extent of the anisotropy and the stimulus field diameter: the data for Subjects A and B indicate a possible proportional relationship, whereas the third subject's data do not.

The findings for vertical correlation confirm earlier measurements by Jenkins (1983b) of the size of the spatial limit using textures of this type for field diameters of $2.5^{\circ}, 5.0^{\circ}$, and $7.5^{\circ}$ of visual angle. In addition, these data indicate the existence of an orientational anisotropy in the detection of correlation. In particular, they illustrate that the spatial limit for the detection of correlation is larger in the vertical orientation.

The expectation on the basis of past findings (see Appelle, 1972) is for vertical and horizontal to be approximately equal, but superior to oblique. In this case, however, vertical is superior to horizontal and oblique,

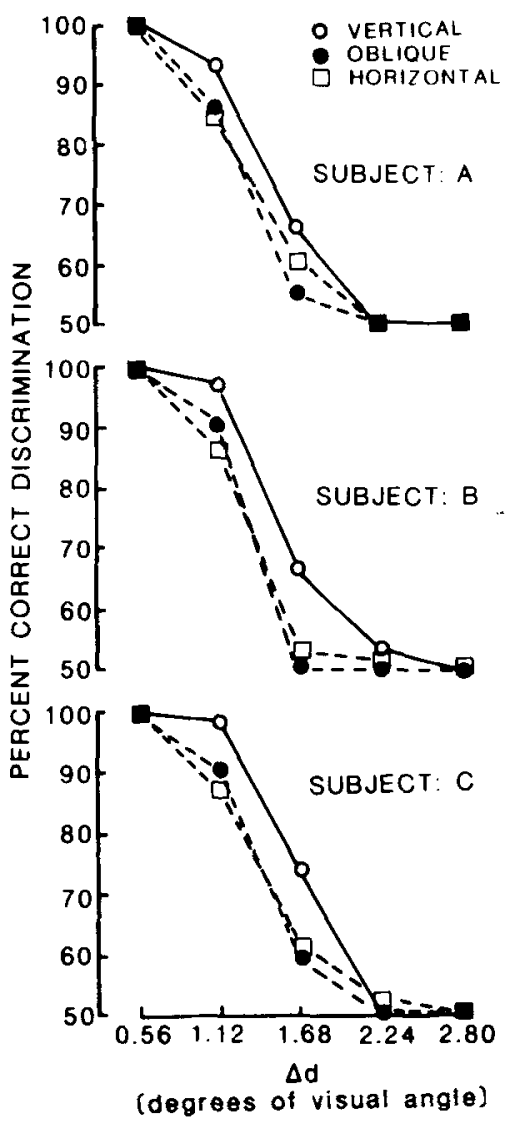

Figure 2. Results of Experiment 1. Percent correct discrimination is plotted against $\Delta d$ (within-point-pair separation) for three orientations (vertical, horizontal, and oblique) for three subjects. Stimulus field diameter is $5^{\circ}$ of visual angle. The size of the data points corresponds to the largest obtained standard error.

with the latter two orientations providing essentially equal spatial limits.

This discrepancy between current and previous findings could reflect the possibility that the anisotropy underlying the detection of correlation is due to factors substantially different from those underlying other visual phenomena. It is also possible that the information concerning relative orientational sensitivity is biased in many previous studies (see Bradshaw, Bradley, \& Patterson, 1976, for a complete discussion of problems in this regard). In the experiments reported here, the sensitivity of each orientation was measured independently of every other orientation, with each being compared to a null stimulus, an equally dense uncorrelated dynamic dot texture. Whatever the difference may be due to, the superior sensitivity to vertical over oblique is a common factor that requires explanation.

\section{EXPERIMENT 2: ORIENTATIONAL ANISOTROPY AND ORIENTATIONAL UNIFORMITY}

If a dynamic transpositionally symmetric texture is constructed by the rapid plotting of identical point-pairs, with 


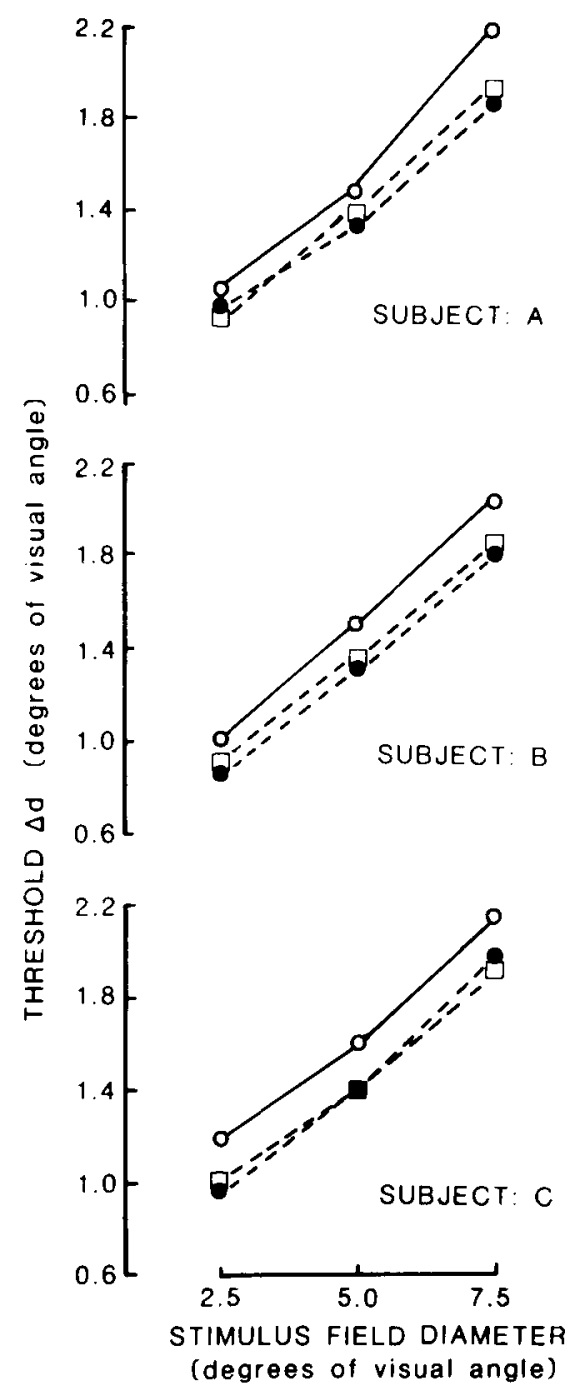

Figure 3. Thresholds for the detection of correlation are plotted against stimulus field diameter, for three orientations, vertical (hollow circle), horizontal (hollow square), and oblique (solid circle) and three subjects. Threshold values correspond to $75 \%$ correct discrimination. The size of the data points corresponds to the largest standard error obtained.

uniform $\Delta d$ and uniform orientation, the visual system can detect the correlation. But if $\Delta d$ is extended beyond a particular value of $\Delta d$, then the detection of correlation breaks down (Jenkins, 1983b). The value of $\Delta d$ corresponding to the threshold between detecting and not detecting the uniformity varies as a function of the uniform orientation (see Experiment 1).

A similar breakdown in detectability of the uniform structure in similar static patterns that occurs as a result of the perturbation of the orientational uniformity, $\Delta \mathrm{p}$, has also been reported (e.g., Glass \& Switkes, 1976). However, these investigators kept $\Delta d$ constant; given that $\Delta d$ has been shown to be a crucial variable in the detection of correlation, as is the axis of uniform orientation, the aim of Experiment 2 was to measure the tolerance for the perturbation of orientational uniformity as a function of several different values of $\Delta d$, along three axes of uniform orientation: vertical, horizontal, and oblique.

\section{Method}

The aim of Experiment 2 was to measure the effect of perturbing orientational uniformity on the detection of correlation, for three orientations (vertical, horizontal, and oblique), for two different stimulus field diameters $(5.0$ and $7.5 \mathrm{~cm})$, and for several values of $\Delta \mathrm{d}\left(7.5-\mathrm{cm}\right.$ diameter $-0.7^{\circ}, 0.9^{\circ}, 1.1^{\circ}, 1.3^{\circ}, 1.5^{\circ}, 1.7^{\circ}$, and $1.9^{\circ}$ of visual angle; $5.0-\mathrm{cm}$ diameter $-0.7^{\circ}, 0.9^{\circ}, 1.1^{\circ}$, and $1.3^{\circ}$ of visual angle). The threshold value of $\Delta p$ was measured independently for each value of $\Delta d$. Within each session, a value of $\Delta \mathrm{d}$ was selected and kept constant; this variable was blocked. Five values of $\Delta p$ were selected. Each session comprised 100 trials, 20 trials of each value of $\Delta \mathrm{p}$. These were presented randomly. The subjects were aware of the axis of orientational uniformity. Five sessions were completed by each subject for each value of $\Delta d$.

Perturbation was achieved by displacing one point of the correlated pair to the left or the right of its true alignment (at random within a given dynamic texture). The varying amounts of increment or decrement, reported here, for technical reasons, as the absolute amount of displacement, $\Delta p$, enabled the orientational uniformity to be perturbed. This method results in a systematic covariation between $\Delta \mathrm{p}$ and $\Delta \mathrm{d}$; however, polar manipulation, which would have avoided this problem, was not possible due to the resolution limits of the display oscilloscope. Accurate compensation of minor variation in $\Delta \mathrm{d}$ was not possible. To limit this technical problem, values of $\Delta d$ were selected that fell within the region of perfect discrimination when $\Delta d$ was the sole variable (see Experiment 1). Any performance variation, therefore, with manipulation of $\Delta p$, was considered to be due to this factor alone.

The subject was presented with two dynamic textures, one a transpositionally symmetric texture, with a particular value of $\Delta \mathrm{d}$ and $\Delta \mathrm{p}$, and an equally dense random-dot texture. Values of $\Delta \mathrm{d}<0.7^{\circ}$ of visual angle were not investigated, since pilot investigations had revealed that orientational pertubation in this case did not result in perceived randomness.

Additional experimental details are contained in the general method; threshold $\Delta \mathrm{p}$ was calculated as for threshold $\Delta \mathrm{d}$.

\section{Results and Discussion}

The results of Experiment 2 are illustrated in Figure 4, and they are plotted as threshold values of $\Delta p$ for three orientations and for two stimulus field diameters. Representative data for one subject are plotted. The other subjects provided data that were qualitatively identical.

The relationship between orientation and threshold $\Delta p$ is complex and is best exemplified by examining the data obtained using the $7.5^{\circ}$-diam stimulus field. An orientational anisotropy is evident for some, but not all, values of $\Delta d$.

For $\Delta d=0.7^{\circ}$ and $0.9^{\circ}$, threshold $\Delta p$ does not vary systematically for the three orientations, and its value appears to be independent of $\Delta \mathrm{d}$. For $\Delta \mathrm{d}=1.1^{\circ}$ and $1.3^{\circ}$, the anisotropy begins to emerge with tolerable $\Delta p$ for the oblique orientation declining rapidly, whereas there is no consistent variation in threshold $\Delta p$ for the vertical and horizontal orientations. For these orientations, threshold $\Delta \mathrm{p}$ remains at approximately the same value as that for the smaller values of $\Delta \mathrm{d}$. For $\Delta \mathrm{d}=1.5^{\circ}$ and $1.7^{\circ}$, the orientational anisotropy is extended to include differences between threshold $\Delta p$ for vertical and horizontal. 


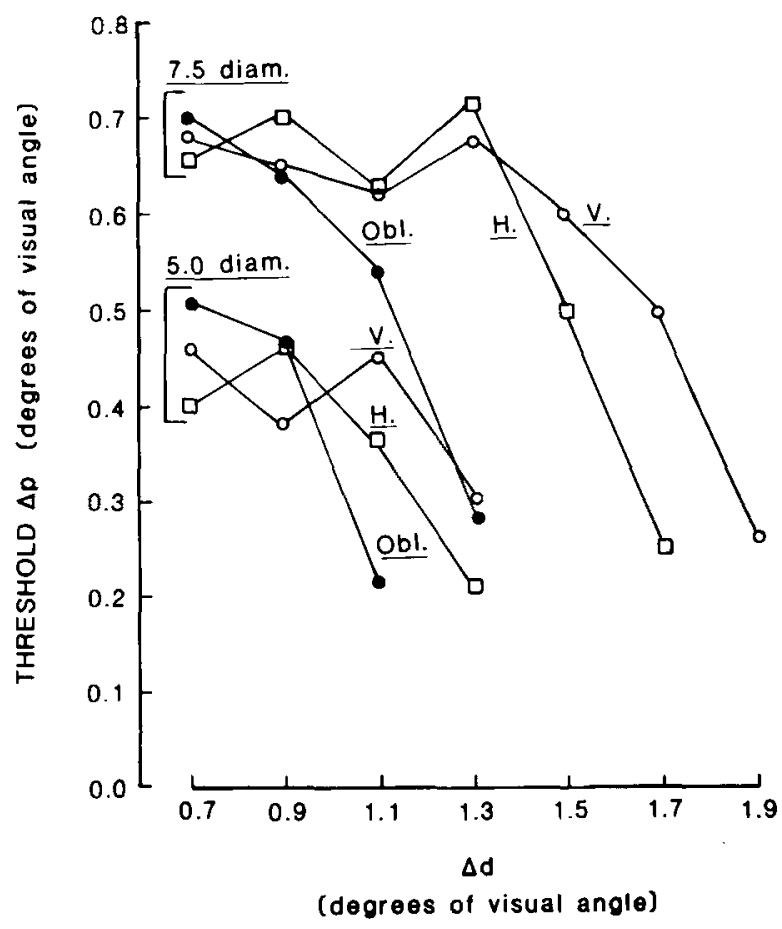

Figure 4. The results of Experiment 2 for Subject $A$ are plotted with threshold $\Delta p$ against $\Delta d$, for three orientations and two field sizes, $5.0^{\circ}$ and $7.5^{\circ}$ diameter. The size of the data points corresponds to the largest standard error.

Confirmation of the reliability of this anisotropy is provided by the fact that qualitatively similar functions were obtained with a $5^{\circ}$-diam field. The difference between the two field sizes with respect to the anisotropy is simply quantitative: the smaller field is associated with smaller values of threshold $\Delta \mathrm{p}$.

It has therefore been shown, in support of previous investigations (Glass \& Switkes, 1976), that perturbing orientational uniformity results in the inability to detect structure. However, the investigation of this threshold over a range of $\Delta d$ values has been justified by the finding that the orientational anisotropy is dependent on the extent of transposition, $\Delta d$.

Focusing on the orientational anisotropy, the question of interest is, "What is unique about the values of $\Delta d$ that demonstrate the anisotropy?" One possible and obvious answer is that the visual system treats them in a fashion that differs qualitatively from symmetric textures with smaller uniform $\Delta d$. This is a possibility, since pilot investigations of the global pattern/randomness threshold revealed a qualitative difference about $\Delta d=0.7^{\circ}$ : perturbation of the orientational uniformity of pairs with $\Delta \mathrm{d}$ $<0.7^{\circ}$ did not result in perceived randomness, no matter what the value of $\Delta \mathrm{p}$. This additional observation of a qualitative distinction adds support for this hypothesis.

A second interesting possibility is that the anisotropy that results from the manipulation of $\Delta p$ is detected only when the detectability of structure is impaired by some other factor; in this case, the obvious candidate is $\Delta \mathrm{d}$.
There is a range of $\Delta d$ values that allow perfect discrimination between transpositionally symmetric textures and equally dense random textures. If the value of $\Delta \mathrm{d}$ falls within this range, then tolerable perturbation is equal and uniform across orientations. However, it may be that as soon as $\Delta d$ extends beyond the value required for perfect discrimination between symmetric and random textures, a more representative index of the true orientational anisotropy is revealed. An argument in support of this hypothesis is that with the manipulation of $\Delta \mathrm{d}$ alone there is a difference in thresholds between vertical, on the one hand, and oblique and horizontal, on the other, with no difference between these latter two orientations. The manipulation of both $\Delta \mathrm{d}$ and $\Delta \mathrm{p}$ results in thresholds that separate quite uniformly and consistently (under two stimulus field sizes) to show quite distinct differences among the three orientations.

Further evidence in support of the second alternative is that the order of sensitivity ranges from vertical to horizontal to oblique. This separation is a better approximation to the anisotropy that is found with other tasks that measure various capacities of the visual system (see Appelle, 1972). This possible explanation is currently being investigated in greater depth to determine whether the exaggerated anisotropy is the direct result of the $\Delta \mathrm{d} \times$ $\Delta p$ interactions or whether it would occur under any $\Delta d$ manipulations with conjoint stimulus impoverishment.

\section{EXPERIMENT 3: ORIENTATIONAL ANISOTROPY AND NUMEROSITY}

Number of elements per unit retinal area, or density, as it is more commonly referred to in the context of symmetric textures, has been reported as being a critical variable by several investigators (e.g., Baker \& Braddick, 1982; Barlow, 1978; Uttal, 1975). For example, in the masking of dot forms by noise, Uttal (1975) has found, not surprisingly perhaps, that if a masking noise-field density is reduced, the detection of the target form improves. Secondly, he has also found that increasing the spacing between the target component dots reduces detectability in noise. Barlow (1978) has investigated density of random-dot patterns as a discriminable dimension, and Baker and Braddick (1982) have investigated the effect of density on the displacement limit for the "short-range" apparent motion in structured dot fields described by Braddick (1974). Jenkins (1983b) has investigated the effect of density on the detectability of correlation at the spatial limit and, like Baker and Braddick (1982), has found no effect of density on these global processes, providing adequate controls are taken. However, these investigations did not examine the relationship between density and orientation; the possibility of an interaction cannot be discarded a priori.

Jenkins (1983b) has reported that texture density has no effect on the spatial limit to the detection of correlation over a wide range $\left(6.5\right.$ to 26 points $\left./ \mathrm{deg}^{2}\right)$. In this 
study, however, the correlation limit was measured for vertical transpositionally symmetric textures only; no other orientation was investigated.

Although texture density has no effect on this limit, the existence of an interaction, as found in the previous experiment, cannot be discounted. The aim of this investigation, therefore, was to investigate the effect of density on the detection of correlation, for three orientations. The variable selected to provide a basis for examining the effect of density was $\Delta d$.

\section{Method}

Experiment 1 was repeated, but with one field size, $5-\mathrm{cm}$ diameter, for three texture densities. Even variation in density of dynamic textures was achieved by manipulating the plotting rate. The three plotting rates used resulted in three apparent texture densities of 26,12 , and 6.5 points $/ \mathrm{deg}^{2}$. The experimental procedure is described in the general method, and was exactly the same as Experiment 1.

\section{Results and Discussion}

The results of Experiment 3 are illustrated in Figure 5 for three subjects, and it can be seen that there is no consistent variation in the spatial limit as a function of orientation, over a fourfold variation in density. It should also be noted that the density of the texture had no effect on the absolute detectability of correlation, therefore confirming both the limit measured here in Experiment 1 and the limit and the effect of density previously measured by Jenkins (1983b).

At each texture density, the vertical spatial limit is greater than both the horizontal and oblique limit, and there is no consistent difference between these latter two orientations. These results suggest that the number of elements in the texture has no bearing on the absolute detectability of structures. If the number of elements has no effect on the absolute limit to the detection of correlation, then it is improbable, by not impossible, that the difference in tolerable $\Delta d$ as a function of orientation is due to the number of elements stimulated. This conclusion does, of course, assume an isomorphism that may not exist.

No interaction between texture density and orientation appears to exist, and, as a result, these data cannot assist in the determination of the basis for the interaction between $\Delta \mathrm{p}$ and orientation illustrated in Experiment 2. The lack of variation in the absolute limit to detectability indicates that decreasing the density may not be an impoverishment; if this is the case, no interaction would be expected to emerge.

\section{GENERAL DISCUSSION}

Using the detectability of correlation in transpositionally symmetric textures as an index of the visual system's sensitivity, the aim of this investigation was to determine whether an orientational anisotropy is carried by the independent texture dimensions of element size, orientational uniformity, and numerosity. The results of the three

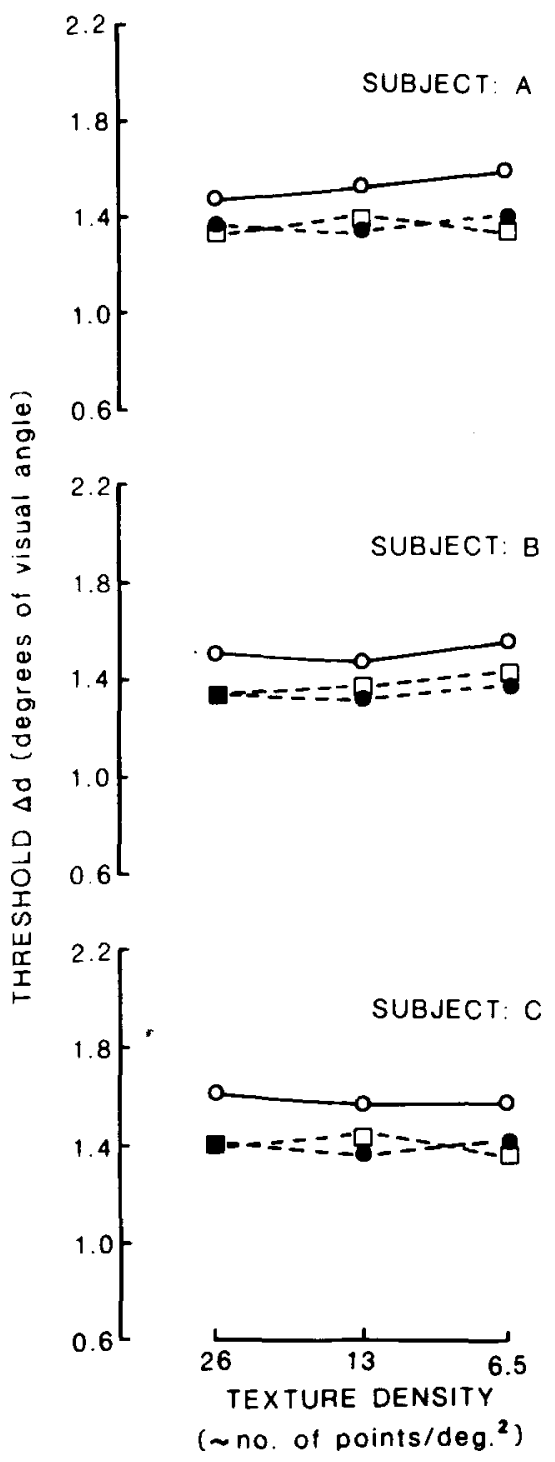

Figure 5. The results of Experiment 3 are plotted for three subjects with threshold $\Delta d$ measured for three texture densities, 6.5, 13, and 26 points/deg ${ }^{2}$, for three orientations: vertical (hollow circle), horizontal (hollow square), and oblique (solid circle). The size of the data points corresponds to the largest obtained standard error.

experiments have shown that, with respect to size, the visual system is capable of detecting correlation carried by larger pairs in the vertical than in the horizontal or the oblique. They have also demonstrated that perturbing the orientational uniformity has no differential effect as a function of orientation, unless the size of the elements is beyond a critical value. This critical value, however, varies as a function of orientation; it is smallest for oblique point-pair patterns, and then increases for horizontal and further again for vertical patterns. There is a clear differentiation between all three orientations in this interaction between $\Delta d$ and $\Delta p$, unlike the previous case. Variation in texture element numerosity has been shown to have no effect on the absolute index of detectability across 
orientations, neither does it enhance any relative difference, as a function of orientation, in this index.

Further, it was found that increasing the stimulus field size increased the absolute detectability of the correlation in the symmetric textures, and this may be attributed, at least tentatively, to the size of the area stimulated, since variation in number of elements, a natural concomitant (see Experiment 3), did nothing to affect the threshold $\Delta$ d. A possible interaction cannot be ruled out. Increasing the field size, however, did nothing to alter the relative variation in threshold $\Delta \mathrm{d}$ as a function of orientation. It is not possible, on examining the data available (see Figure 3), to determine whether there is an absolute difference in the relative variation as a function of field size or whether the relative difference is a constant proportion. Further investigation may clarify this issue.

It is perhaps unnecessary to point out that of the visual processes of a fundamental nature that could carry an orientational anisotropy, the integration of information over retinal space is just one. Additional processes include temporal integration and spatial differentiation. In addition, most of the phenomena that exhibit the orientational anisotropy rely not only upon a combination of the above processes, but also on cognitive and judgmental processes. It is possible that further anisotropies exist at each of these higher levels also. It may be that any given net anisotropy is the result of the operation of any or all anisotropies that exist at many different levels.

However, given that Atkinson's (1972) hypotheses were responsible for the identification of the three variables of interest in relation to the orientational anisotropy, it may be constructive to speculate on the extent to which the data obtained in these experiments can be accounted for by reference to functional units with elongated receptive fields in the human visual system.

The data cannot elaborate on the "numerosity" hypothesis, since psychophysical data cannot be considered as evidence where specific cell counts are required. However, with respect to the other two hypotheses, the data may contribute.

The implication of the data with respect to these two hypotheses can best be examined if isosensitivity curves are constructed on the basis of the data obtained in Experiments 1 and 2 (see Figure 6). If these two dimensional spaces can be viewed as mapping the spatial bounds of each identical point-pair element, inferred from the measured limits of the population of such elements, then several characteristics may be pertinent to the two hypotheses. It is obvious, on inspecting Figure 6, that, although there are slight variations in shape as a function of orientation, the most salient comparison is in the relative extent of the isosensitivity spaces; vertical is longer than horizontal and oblique. If the data are viewed in this fashion, and the two-dimensional space is assumed to represent the length and breadth of the receptive fields serving functional units, then the data support the "extent" hypothesis but not the "selectivity" hypothesis.

Finding a larger tolerable spatial extent for vertical

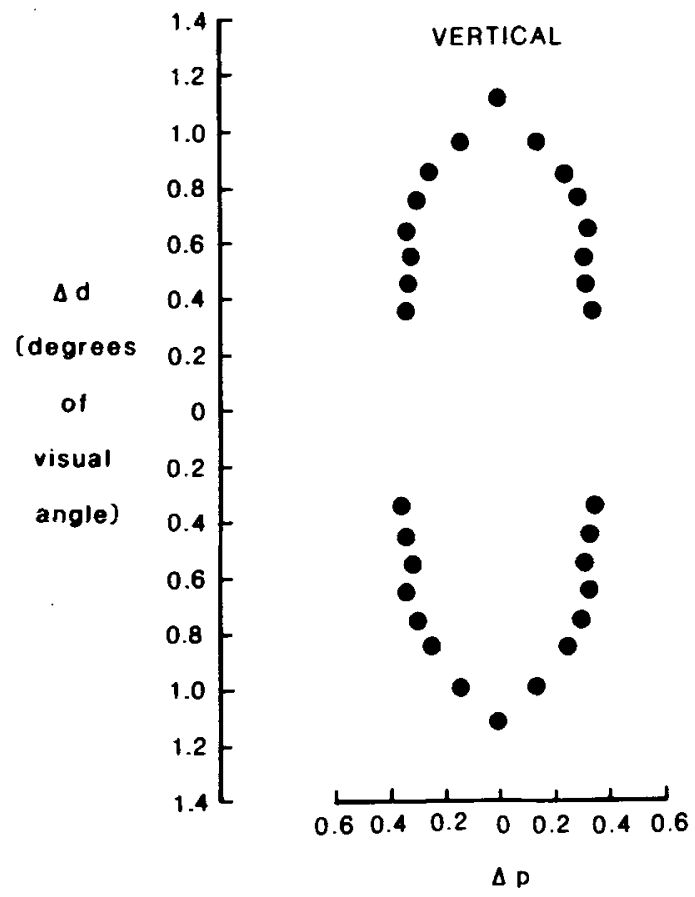

(degrees of visual angle)
HORIZONTAL
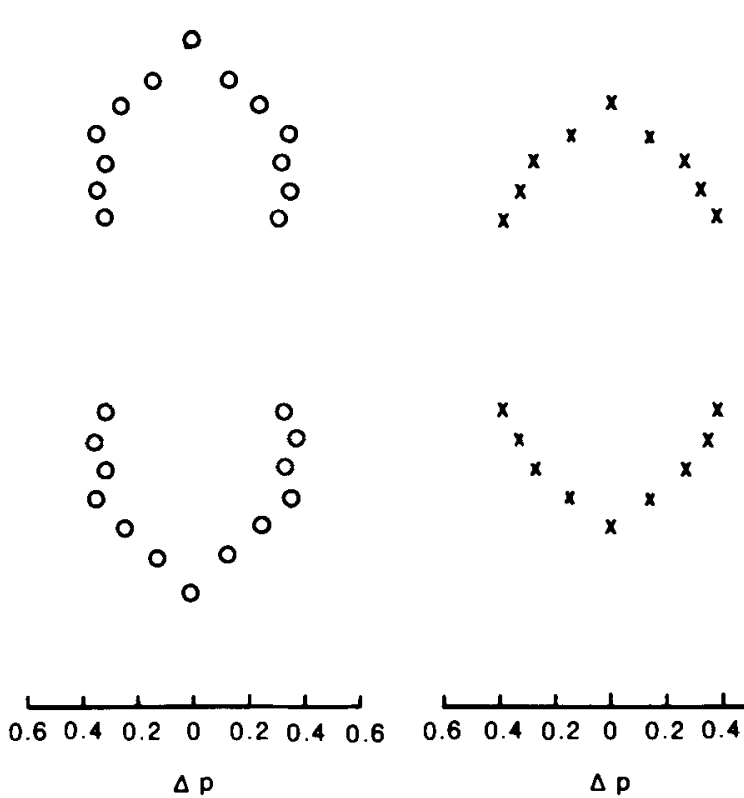

(degrees of visual angle)

\section{OBLIQUE}
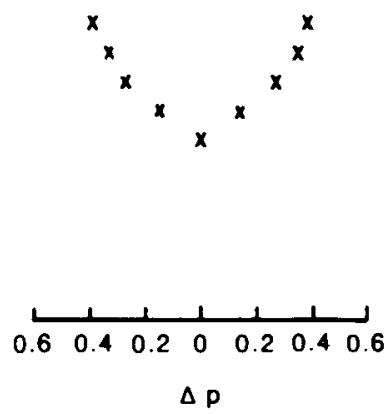

(degrees of visual angle)

Figure 6. Information provided by Experiments 1 and 2, for Subject A, is used to construct two-dimensional spatial isosensitivity profites for the detection of correlation, for three orientations. 
correlation is novel; but accounting for the data by postulating longer functional unit receptive fields in the vertical raises many questions. It is useful to consider the "size" hypothesis as accounting for the obtained data, but the most obvious question remains: How can such a structural characteristic account for vertical sensitivity in general? The problems raised by such a question draw attention to the proposal earlier in this discussion that orientational anisotropies may exist at all levels of the visual process, and that more than one anisotropy may exist in one level.

Additional questions that the data raise relate to the functional importance of the orientational anisotropy to vision in general and to the detection of correlation in particular. With respect to the former, the argument has been expressed that we are more sensitive to verticals because of the predominance of verticality in our environment (e.g., Annis \& Frost, 1973), and that therefore the organism is advantaged if it is sensitive to verticals. This argument highlights several problems, but one in particular is of relevance to this discussion, and that is the implicit assumption that greater spatial integration or sensitivity in the vertical meridian is advantageous. This may not be the case, however, and it seems less than cautious to attribute functional gain to a visual process simply because of its quantitative value, relative or absolute.

This point can be further illustrated in the specific case of the detection of correlation. If the correlation mechanism can operate over a larger spatial range at the retina vertically than horizontally or obliquely, does this mean that detecting correlations vertically is more critical than detecting them in other orientations? The problem highlighted here is that unless the functions of identified processes or mechanisms are known, imbuing measured differences with functional value is hazardous.

The human visual system's sensitivity to correlation in transpositionally symmetric textures was measured using the global pattern/randomness threshold as an index of this sensitivity. These thresholds were found to be dependent on the size of the point-pair element, where the tolerable size varied as a function of orientation, with the system allowing a larger tolerable $\Delta \mathrm{d}$ in the vertical meridian. Increasing the stimulus field size increased the absolute value of this threshold, but not the relative value as a function of orientation. The thresholds were also found to be dependent on the orientational uniformity of the pointpairs. Tolerable perturbation did not vary as a function of the uniform orientation until a critical point-pair size was exceeded. This critical point-pair size did vary as a function of orientation, falling quite distinctly from vertical, to horizontal, to oblique. The number of point-pair elements comprising the textures did not affect the global pattern/randomness threshold.

\section{REFERENCES}

ANNIS, R. C., \& Frost, B. C. (1973). Human visual ecology and orientation anisotropies in acuity. Science, 182, 729-731.
Anton, B. S. (1976). Poggendorff illusion as a function of orientation of transversals and parallel lines. Perceptual and Motor Skills, 43, 83-90.

APPELLE, S. (1972). Perception and discrimination as a function of stimulus orientation: The "oblique effect" in man and animals. Psychological Bulletin, 78, 266-278.

APPELLE, S. (1976). Figure embeddedness depends on contour orientation. Perception \& Psychophysics, 19, 109-112.

AtKInson, J. (1972). The effect of size, retinal locus, and orientation on the visibility of a single afterimage. Perception \& Psychophysics, 12, 213-217

BAKER, C. L., \& Braddick, O. J. (1982). The basis of area and dot number effects in random dot motion perception. Vision Research, 22, 1253-1259.

BARLOW, H. B. (1978). The efficiency of detecting changes of density in random dot patterns. Vision Research, 18, 637-650.

Blakemore, C. B., Carpenter, R. H. S., \& Georgeson, M. A. (1970). Lateral inhibition between orientation detectors in the human visual system. Nature, 228, 37-39.

Blakemore, C. B., \& NACHMias, J. (1971). The orientational specificity of two visual after-effects. Journal of Physiology (London), 213, 157-174.

Blakemore, C. B., Nachmias, J., \& Sutton, P. (1970). The perceived spatial frequency shift: Evidence for frequency selective neurons in the human brain. Journal of Physiology (London), 210, 727-750.

BRADDICK, O. J. (1974). A short range process in apparent motion. Vision Research, 14, 519-527.

Braddick, O. J., Campbell, F. W., \& Atkinson, J. (1978). Channels in vision: Basic aspects. In R. Held, H. Leibowitz, \& H. L. Teuber (Eds.), Handbook of sensory physiology (Vol. VIII): Perception. Heidelburg: Springer-Verlag.

Bradshaw, J., Bradley, D. \& Patterson, K. (1976). The perception and identification of mirror-reversed patterns. Quarterly Journal of Experimental Psychology, 28, 221-246.

CAMPBELl, F. W., \& KulikowsKI, J. J. (1966). Orientation selectivity in the human visual system. Journal of Physiology (London), 187, 437-445.

Campbell, F. W., Kulikowski, J. J., \& Levinson, J. (1966). The effect of orientation on the visual resolution of gratings. Journal of Physiology (London), 187, 427-436.

CAMPBell, F. W., \& MAFFEI, L. (1970). Electrophysiological evidence for the existence of orientation and size detectors in the human visual system. Journal of Physiology (London), 207, 635-652.

EMSLEY, H. H. (1925). Irregular astigmatism of the eye: Effect of correcting lenses. Transactions of the Optical Society, 27, 28-42.

Freeman, R. D., \& Thibos, L. N. (1973). Electrophysiological evidence that abnormal early visual experience can modify the human brain. Science, 180, 876-878.

FURCHNER, C. S., \& YOUNG, S. J. (1975). Recovery from adaptation as a function of stimulus orientation. Perception \& Psychophysics, $17,117-124$

GiBson, J. J. (1933). Adaptation, after-effect and contrast in perception of curved lines. Joumal of Experimental Psychology, 16, 1-31.

GiLINSKY, A. S., \& MAYO, T. H. (1971). Inhibitory effects of orientational adaptation. Journal of the Optical Society of America, 61, $1710-1714$

GLass, L. (1969). Moiré effect from random dots. Nature, 223, 578-580.

Glass, L., \& Perez, R. (1973). Perception of random dot interference patterns. Nature, 246, 360-362.

Glass, L., \& SwTtKes, E. (1976). Pattern recognition in humans: Correlations which cannot be perceived. Perception, 5, 67-72.

Green, R. T., \& Hoyle, E. M. (1964). The influence of spatial orientation on the Poggendorf illusion. Acta Psychologica, 22, 348-366.

Gregory, R. L. (1972). Cognitive contours. Nature, 238, 51-52.

Houlhan, K., \& Sekuler, R. W. (1968). Contour interaction in visual masking. Journal of Experimental Psychology, 77, 281-285.

Hubel, D. H., \& Wiesel, T. N. (1977). Ferrier lecture: Functional architecture of macaque monkey visual cortex. Proceedings of the Royal Society, London, Series B, 198, 1-59.

JASTROW, J. (1892). On the judgement of angles and positions of lines. American Journal of Psychology, 5, 214-248. 
JENKINS, B. (1982). Redundancy in the perception of bilateral symmetry in dot textures. Perception \& Psychophysics, 32, 171-177.

JENKINS, B. (1983a). Component processes in the perception of bilaterally symmetric dot textures. Perception \& Psychophysics, 34, 433-440.

JENKINS, B. (1983b). Spatial limits to the detection of transpositional symmetry in dynamic dot textures. Journal of Experimental Psychology: Human Perception and Performance, 9, 258-269.

JENKINS, B. (1983c). Temporal limits to the detection of correlation in transpositionally symmetric textures. Perception \& Psychophysics, 33, 79-84.

JuLESZ, B. (1971). Foundations of cyclopean perception. Chicago and London: University of Chicago Press.

KitTerle, F. L., Russell, S. K., \& Nixon, H. (1974). Pattern alternation: Effects of spatial frequency and orientation. Perception \& Psychophysics, 16, 543-546.

KOHLER, W., \& WALLACH, H. (1944). Figural after-effects: An investigation of visual processes. Proceedings of the American Philosophical Society, 88, 269-357.

LEIBOWITZ, H. W. (1955). Some factors influencing the variability of vernier adjustments. American Journal of Psychology, 68, 266-273.

MAFFEI, L., \& CAMPBELL, F. W. (1970). Neurophysiological localiza- tion of the vertical and horizontal visual coordinates in man. Science, 167, 386-387.

MORGAN, M. J., \& WARD, R. (1980). Conditions for motion flow in dynamic visual noise. Vision Research, 20, 431-435.

Movshon, J. A., BlaKemore, C. B. (1973). Orientational specificity and spatial selectivity in human vision. Perception, 2, 53-60.

PARLeE, M. B. (1969). Visual background masking of a single line by a single line. Vision Research, 9, 199-205.

Rochlin, A. M. (1955). The effect of tilt on the visual perception of parallelness. American Journal of Psychology, 68, 223-236.

SeKUler, R. W. (1965). Spatial and temporal determinants of visual backward masking. Journal of Experimental Psychology, 70, 401-406.

SUTHERLAND, N. S. (1957). Visual discrimination of orientation by octopus. British Journal of Psychology, 48, 55-71.

UTTAL, W. R. (1975). An autocorrelational theory of form detection, Hillsdale, NJ: Erlbaum.

(Manuscript received May 10, 1984; revision accepted for publication January 4, 1985.) 